\title{
SUFFICIENT CONDITIONS FOR OSCILLATIONS OF ALL SOLUTIONS OF A CLASS OF IMPULSIVE DIFFERENTIAL EQUATIONS WITH DEVIATING ARGUMENT
}

\author{
D.D. BAINOV \\ Medical University \\ P.O. Box 45 \\ Sofia 1504 Bulgaria \\ M.B. DIMITROVA \\ Technical University \\ Sliven 8800, Bulgaria
}

(Received January, 1994; Revised September, 1995)

\begin{abstract}
Sufficient conditions are found for oscillation of all solutions of impulsive differential equation with deviating argument.
\end{abstract}

Key words: Oscillation, Impulsive Differential Equations.

AMS (MOS) subject classifications: $34 \mathrm{~A} 37$.

\section{Introduction}

The impulsive differential equations with deviating argument are adequate mathematical models of numerous processes and phenomena in physics, biology and electrical engineering. In spite of wide possibilities for their application, the theory of these equations is developing rather slowly because of considerable difficulties of technical and theoretical character related to their study.

In the recent twenty years, the number of investigations devoted to the oscillatory and nonoscillatory behavior of the solutions of functional differential equations has considerably increased. The large part of the works on this subject published by 1977 is presented in [4]. In monographs [2] and [3], published in 1987 and 1991, respectively, the oscillatory and asymptotic properties of the solutions of various classes of functional differential equations were systematically studied. A pioneering work devoted to the investigation of the oscillatory properties of the solutions of impulsive differential equations with deviating argument was rendered by Gopalsamy and Zhang [1].

In the present paper, sufficient conditions are found for oscillation of all solutions of the equation

$$
\begin{gathered}
x^{\prime}(t)-p(t) x(t+h)=0, \quad t \neq \tau_{k}, \\
\Delta x\left(\tau_{k}\right)=x\left(\tau_{k}+0\right)-x\left(\tau_{k}-0\right)=b_{k} x\left(\tau_{k}-0\right)=b_{k} x\left(\tau_{k}\right)
\end{gathered}
$$


where the function $p=p(t)$ is nonnegative and continuous, and $\tau_{k}(k \in \mathbb{N})$ are fixed moments of impulsive effect.

\section{Preliminary Notes}

Let $\mathbb{N}_{n}=\{1,2, \ldots, n\}, p \in C\left(\mathbb{R}_{+}, \mathbb{R}_{+}\right), \mathbb{R}_{+}=[0, \infty)$, let $h$ be a positive constant, $\left\{\tau_{k}\right\}_{k=1}^{\infty}$ be a monotone increasing, unbounded sequence of real numbers, and $\left\{b_{k}\right\}_{k=1}^{\infty}$ be a sequence of real numbers.

Consider the impulsive differential equation with a deviating argument (1) under the condition

$$
x(t)=\varphi(t), \quad t \in[0, h)
$$

where $\varphi \in C^{1}\left([0, h), \mathbb{R}_{+}\right)$.

Introduce the following conditions:

H1: $\quad 0<h<\tau_{1}$.

H2: There exists a positive constant $T>h$ such that $\tau_{k+1}-\tau_{k} \geq T, k \in \mathbb{N}$.

H3: There exists a constant $M>0$ such that for any $k \in \mathbb{N}$ the inequality $0 \leq M \leq b_{k}$ is valid.

We construct the sequence

$$
\left\{t_{k}, k \in \mathbb{N}\right\}=\left\{\tau_{k}, k \in \mathbb{N}\right\} \cup\left\{\tau_{k}-h, k \in \mathbb{N}\right\}
$$

so that $t_{k}<t_{k+1}, k \in \mathbb{N}$.

Definition 1: By a solution of equation (1) under condition (2) we mean any function $x:[0, \infty) \rightarrow \mathbb{R}$ for which the following holds true:

1. If $0 \leq t \leq t_{1}=\tau_{1}-h$, then the function $x$ coincides with the solution of the problem

$$
x^{\prime}(t)-p(t) x(t+h)=0
$$

2. If $t_{k}<t \leq t_{k+1}, t_{k} \in\left\{\tau_{k}, k \in \mathbb{N}\right\} \backslash\left\{\tau_{k}-h, k \in \mathbb{N}\right\}$, then the function $x$ coincides with the solution of the problem

$$
\begin{gathered}
x^{\prime}(t)-p(t) x(t+h)=0 \\
x\left(t_{k}+0\right)=\left(1+b_{k_{i}}\right) x\left(t_{k}\right),
\end{gathered}
$$

where $k_{i}$ is determined from the equality $\tau_{k_{i}}=t_{k}$.

3. If $t_{k}<t \leq t_{k+1}, t_{k} \in\left\{\tau_{k}-h, k \in \mathbb{N}\right\} \backslash\left\{\tau_{k}, k \in \mathbb{N}\right\}$, then the function $x$ coincides with the solution of the problem

$$
\begin{gathered}
x^{\prime}(t)-p(t) x(t+h+0)=0 \\
x\left(t_{k}+0\right)=x\left(t_{k}\right) .
\end{gathered}
$$

4. If $t_{k}<t \leq t_{k+1}, t_{k} \in\left\{\tau_{k}, k \in \mathbb{N}\right\} \cap\left\{\tau_{k}-h, k \in \mathbb{N}\right\}$, then the function $x$ coincides with the solution of the problem 


$$
\begin{gathered}
x^{\prime}(t)-p(t) x(t+h+0)=0 \\
x\left(t_{k}+0\right)=\left(1+b_{k_{i}}\right) x\left(t_{k}\right),
\end{gathered}
$$

where $k_{i}$ is determined from the equality $\tau_{k_{i}}=t_{k}$.

Definition 2: A nonzero solution $x$ of equation (1) is said to be nonoscillating if there exists $t_{0} \geq 0$ such that $x(t)$ is of constant sign for $t \geq t_{0}$. Otherwise, the solution $x$ is said to oscillate.

\section{Main Results}

Theorem 1: Let the following conditions hold:

1. Conditions $\mathrm{H} 1$ and $\mathrm{H} 2$ are met.

2. $\quad \limsup _{i \rightarrow \infty}\left(1+b_{i}\right) \int_{\tau_{i}-h}^{\tau_{i}} p(s) d s>1$.

Then all solutions of equation (1) oscillate.

Proof: Let a nonoscillating solution $x$ of equation (1) exist. Without loss of generality we assume that $x(t)>0$ for $t \geq t_{0}>0$. Then $x(t+h)>0$ also for $t \geq t_{0}$.

From (1), it follows that $x$ is a nonincreasing function in $\left(t_{0}, \tau_{k}\right) \cup\left[\cup_{i=k}^{\infty}\left(\tau_{i}, \tau_{i+1}\right)\right]$, where $\tau_{k}>t_{0}>\tau_{k-1}$.

Integrate (1) from $\tau_{i}-h$ to $\tau_{i}(i \geq k+1)$ and obtain

Since

$$
\begin{gathered}
x\left(\tau_{i}\right)-x\left(\tau_{i}-h\right)=\int_{\tau_{i}-h}^{\tau_{i}} p(s) x(s+h) d s, \\
x\left(\tau_{i}\right)-x\left(\tau_{i}-h\right) \geq x\left(\tau_{i}+0\right) \int_{\tau_{i}-h}^{\tau_{i}} p(s) d s .
\end{gathered}
$$

$$
x\left(\tau_{i}+0\right)=\left(1+b_{i}\right) x\left(\tau_{i}-0\right)=\left(1+b_{i}\right) x\left(\tau_{i}\right)
$$

then (3) and (4) yield the inequality

$$
x\left(\tau_{i}-h\right)+x\left(\tau_{i}\right)\left[\left(1+b_{i}\right) \int_{\tau_{i}-h}^{\tau_{i}} p(s) d s-1\right] \leq 0 .
$$

Inequality (5) is valid only if

$$
\limsup _{i \rightarrow \infty}\left(1+b_{i}\right) \int_{\tau_{i}-h}^{\tau_{i}} p(s) d s \leq 1,
$$

which contradicts condition 2 of Theorem 1.

Theorem 2: Let the following conditions hold: 


\section{Conditions H1-H3 are met.}

2. $\quad \liminf _{t \rightarrow \infty} \int_{t}^{t+h} p(s) d s>\frac{1}{e(1+M)}$.

Then all solutions of equation (1) oscillate.

Proof: Let a nonoscillating solution $x$ of equation (1) exist. Without loss of generality we assume that $x(t)>0$ for $t \geq t_{0}>0$. Then $x(t+h)>0$ also for $t \geq t_{0}$.

From (1) it follows that $x$ is a nondecreasing function in $\left(t_{0}, \tau_{k}\right) \cup\left[\cup_{i=k}^{\infty}\left(\tau_{i}, \tau_{i+1}\right)\right]$, $\tau_{k-1}<t_{0}<\tau_{k}$.

Define the function $w(t)=\frac{x(t+h)}{x(t)}, t \geq t_{0}$, and let $\tau_{i} \in(t, t+h), t \geq t_{0}$. Then

$$
x(t) \leq x\left(\tau_{i}\right)=\frac{x\left(\tau_{i}+0\right)}{1+b_{i}} \leq \frac{x(t+h)}{1+b_{i}} \leq \frac{x(t+h)}{1+M} .
$$

From the last inequality it follows that $w(t) \geq 1+M$ for $t \geq t_{0}$.

We shall prove that the function $w$ is bounded from above for $t \geq t_{0}$.

1. Let $\tau_{i} \in\left(t, t+\frac{h}{2}\right), t \geq t_{0}$. Integrate (1) from $t$ to $t+\frac{h}{2}$ and obtain that

$$
x\left(\tau_{i}\right)-x(t)+x\left(t+\frac{h}{2}\right)-x\left(\tau_{i}+0\right)=\int_{t}^{t+h / 2} p(s) x(s+h) d s .
$$

Since

$$
x\left(\tau_{i}+0\right)=\left(1+b_{i}\right) x\left(\tau_{i}\right)
$$

then from (6) and (7) it follows that

$$
x\left(t+\frac{h}{2}\right)=x(t)+\int_{t}^{t+h / 2} p(s) x(s+h) d s+b_{i} x\left(\tau_{i}\right) .
$$

From (8) we obtain that

$$
x\left(t+\frac{h}{2}\right) \geq \inf _{s \in[t, t+h / 2]} x(s+h) \int_{t}^{t+h / 2} p(s) d s=\inf _{s \in[t+h, t+3 h / 2]} x(s) \int_{t}^{t+h / 2} p(s) d s \text {. }
$$

If in the interval $\left[t+h, t+\frac{3 h}{2}\right]$ there is no point of jump, then

$$
\inf _{s \in[t+h, t+3 h / 2]} x(s)=x(t+h) .
$$

If in the interval $\left[t+h, t+\frac{3 h}{2}\right]$ there is a point of jump, $\tau_{i+1}$, then from the inequalities

$$
x(t+h) \leq x\left(\tau_{i+1}\right)=\frac{x\left(\tau_{i+1}+0\right)}{1+b_{i}} \leq \frac{x\left(t+\frac{3 h}{2}\right)}{1+M}
$$

it follows that

$$
\inf _{s \in[t+h, t+3 h / 2]} x(s)=x(t+h)
$$

The last inequality and (9) lead to

$$
x\left(t+\frac{h}{2}\right) \geq x(t+h) \int_{t}^{t+h / 2} p(s) d s
$$


Integrating (1) from $t+\frac{h}{2}$ to $t+h$, we get

$$
x(t+h)-x\left(t+\frac{h}{2}\right) \geq x\left(t+\frac{3 h}{2}\right) \int_{t+h / 2}^{t+h} p(s) d s .
$$

From (10) and (11) it follows that

$$
\frac{x\left(t+\frac{3 h}{2}\right)}{x\left(t+\frac{h}{2}\right)} \leq \frac{1}{\int_{t}^{t+h / 2} p(s) d s \int_{t+h / 2}^{t+h} p(s) d s} \leq \text { const. }
$$

Thus we proved that the function $w$ is bounded from above.

2. Let $\tau_{i} \in\left(t+\frac{h}{2}, t+h\right)$. The boundedness from above of the function $w$ can be proved analogously.

We divide (1) by $x(t)>0, t \geq t_{0}$, integrate from $t$ to $t+h$ and obtain

$$
\begin{gathered}
\int_{t}^{\tau_{i}} \frac{x^{\prime}(s)}{x(s)} d s+\int_{\tau_{i}}^{t+h} \frac{x^{\prime}(s)}{x(s)} d s=\int_{t}^{t+h} p(s) \frac{x(s+h)}{x(s)} d s \\
\ln \left[\frac{1}{1+b_{i}} w(t)\right]=\int_{t}^{t+h} p(s) w(s) d s .
\end{gathered}
$$

From (12) it follows that

$$
\ln \left[\frac{1}{1+M} w(t)\right] \geq \liminf _{t \rightarrow \infty} w(t) \int_{t}^{t+h} p(s) d s
$$

Denote $w_{0}=\liminf _{t \rightarrow \infty} w(t), 0<w_{0}<\infty$. Then from (13) we obtain

$$
\liminf _{t \rightarrow \infty} \int_{t}^{t+h} p(s) d s \leq \frac{\ln \left[(1+M)^{-1} w_{0}\right]}{w_{0}} \leq \frac{1}{e(1+M)} .
$$

The last inequality contradicts condition 2 of Theorem 2 .

Corollary 1: Let the conditions of Theorem 2 hold. Then:

1. The inequality

$$
\begin{gathered}
x^{\prime}(t)-p(t) x(t+h) \geq 0, \quad t \neq \tau_{k}, \\
\Delta x\left(\tau_{k}\right)=b_{k} x\left(\tau_{k}\right)
\end{gathered}
$$

has no positive solutions.

2. The inequality

$$
\begin{gathered}
x^{\prime}(t)-p(t) x(t+h) \leq 0, \quad t \neq \tau_{k}, \\
\Delta x\left(\tau_{k}\right)=b_{k} x\left(\tau_{k}\right)
\end{gathered}
$$

has no negative solutions. 
Proof of 2: Let inequality (15) have a negative solution $x(t)$ for $t \geq t_{0}$ for some $t_{0} \geq 0$. From (15) it follows that

$$
x^{\prime}(t) \leq p(t) x(t+h) \leq 0,
$$

i.e., $x$ is a nonincreasing function in $\left(t_{0}, \tau_{k}\right) \cup\left[\cup_{i=k}^{\infty}\left(\tau_{i}, \tau_{i+1}\right)\right]$.

From (16) we obtain that

$$
\frac{x^{\prime}(t)}{x(t)} \geq p(t) \frac{x(t+h)}{x(t)}
$$

Analogously to the proof of Theorem 2 we are led to a contradiction with condition 2 of Theorem 2.

Theorem 3: Let the following conditions hold:

1. Conditions H1-H3 are met.

2. $\quad \liminf _{k \rightarrow \infty} \int_{\tau_{k}-h}^{\tau_{k}} p(s) d s>\frac{1}{1+M}$.

Then all solutions of equation (1) oscillate.

Proof: From (3) analogously to the proof of Theorem 1 we obtain

$$
\liminf _{k \rightarrow \infty} \int_{\tau_{k}-h}^{\tau_{k}} p(s) d s \leq \frac{x\left(\tau_{k}\right)}{x\left(\tau_{k}+0\right)}=\frac{1}{1+b_{k}} \leq \frac{1}{1+M} .
$$

The last inequality contradicts condition 2 of Theorem 3 .

Corollary 2: Let the conditions of Theorem 3 hold. Then:

1. Inequality (14) has no positive solutions.

2. Inequality (15) has no negative solutions.

The proof of Corollary 2 is carried out analogously to the proof of Corollary 1.

Theorem 4: Let the following conditions hold:

1. Conditions H1- H3 are satisfied.

2. In each interval of length $h$ there are $k$ points of jump $(k \in \mathbb{N})$.

3. $\quad \liminf _{t \rightarrow \infty} \int_{t}^{t+h} p(s) d s>\frac{1}{e(1+M)^{k}}$.

Then all solutions of equation 1 oscillate.

Proof: Let a nonoscillating solution $x$ of equation (1) exist. Without loss of generality we assume that $x(t)>0$ for $t \geq t_{0}>0$. Then $x(t+h)>0$ also for $t \geq 0$.

For any fixed $t\left(t \geq t_{0}\right)$ in the interval $(t, t+h)$, let

$$
t<\tau_{s}^{(1)}<\tau_{s}^{(2)}<\ldots<\tau_{s}^{(k)}<t+h
$$

be $k$ points of jump with respective constants $b_{s}^{(1)}, b_{s}^{(2)}, \ldots, b_{s}^{(k)}$.

Since $x\left(\tau_{s}\right)=\frac{x\left(\tau_{s}+0\right)}{1+b_{s}}, s \in \mathbb{N}$ and $x$ is a nondecreasing function in $\left(t, \tau_{s}^{(1)}\right) \cup$

$\left[\cup_{i=1}^{k-1}\left(\tau_{s}^{(i)}, \tau_{s}^{(i+1)}\right)\right] \cup\left(\tau_{s}^{(k)}, t+h\right)$, then 


$$
x(t) \leq x\left(\tau_{s}^{(1)}\right)=\frac{x\left(\tau_{s}^{(1)}+0\right)}{1+b_{s}^{(1)}} \leq \ldots \leq \frac{x(t+h)}{\prod_{i=1}^{k}\left(1+b_{s}^{(i)}\right)} .
$$

From (17) it follows that

$$
\frac{x(t+h)}{x(t)} \geq(1+M)^{k}
$$

Introduce the function $w(t)=\frac{x(t+h)}{x(t)}, t \geq t_{0}$.

We shall prove that the function $w$ is bounded from above for $t \geq t_{0}$.

Let the interval $\left[t, t+\frac{h}{2}\right]$ contain $l$ points of jumps, and let the interval $\left[t+\frac{h}{2}, t+h\right]$ contain $r$ points of jumps $(l+r=k)$.

Integrate (1) from $t$ to $t+\frac{h}{2}$ and obtain that

$$
x\left(t+\frac{h}{2}\right)-x(t)=\int_{t}^{t+h / 2} p(s) x(s+h) d s+\sum_{i=1}^{l} b_{s}^{(i)} x\left(\tau_{s}^{(i)}\right) .
$$

From (18) it follows that

$$
x\left(t+\frac{h}{2}\right) \geq x(t+h) \int_{t}^{t+h / 2} p(s) d s .
$$

Integrate (1) from $t+\frac{h}{2}$ to $t+h$ and obtain that

$$
x(t+h)-x\left(t+\frac{h}{2}\right)=\int_{t+h / 2}^{t+h} p(s) x(s+h) d s+\sum_{i=l+1}^{k} b_{s}^{(i)} x\left(\tau_{s}^{(i)}\right) .
$$

From (20) it follows that

$$
x(t+h) \geq x\left(t+\frac{3 h}{2}\right) \int_{t}^{t+h} p(s) d s .
$$

From (19) and (21) we obtain that

$$
\frac{x\left(t+\frac{3 h}{2}\right)}{x\left(t+\frac{h}{2}\right)} \leq \frac{1}{\int_{t}^{t+h / 2} p(s) d s \int_{t+h / 2}^{t+h} p(s) d s} \leq \text { const. }
$$

From the last inequality it follows that the function $w$ is bounded from above for $t \geq t_{0}$.

Denote $w_{0}=\liminf _{t \rightarrow \infty} w(t), 0<w_{0}<\infty$.

Integrate

$$
\frac{x^{\prime}(t)}{x(t)}-p(t) \frac{x(t+h)}{x(t)}=0
$$

from $t$ to $t+h, t \geq t_{0}$, and obtain

$$
\ln \frac{x(t+h)}{x(t)}+\sum_{i=1}^{k}\left[\ln x\left(\tau_{s}^{(i)}\right)-\ln x\left(\tau_{s}^{(i)}+0\right)\right]=\int_{t}^{t+h} p(s) \frac{x(s+h)}{x(s)} d s
$$




$$
\ln \left[\frac{w(t)}{\prod_{i=1}^{k}\left(1+b_{s}^{(i)}\right)}\right]=\int_{t}^{t+h} p(s) w(s) d s
$$

Assertion (22) leads to the inequality

$$
\ln \left[\frac{w(t)}{(1+M)^{k}}\right] \geq \liminf _{t \rightarrow \infty} w(t) \int_{t}^{t+h} p(s) d s .
$$

From the last inequality we obtain that

$$
\liminf _{t \rightarrow \infty} \int_{t}^{t+h} p(s) d s \leq \frac{\ln \left[(1+M)^{-k} w_{0}\right]}{w_{0}} \leq \frac{1}{e(1+M)^{k}}
$$

which contradicts condition 3 of Theorem 4.

Corollary 3: Let the conditions of Theorem 4 hold. Then:

1. Inequality (14) has no positive solutions.

2. Inequality (15) has no negative solutions.

The proof of Corollary 3 can be rendered analogously to the proof of Corollary 1 and Theorem 4.

Consider the nonhomogeneous impulsive differential equation with deviating argument:

$$
\begin{gathered}
x^{\prime}(t)-p(t) x(t+h)=q(t), \quad t \neq \tau_{k}, \\
\Delta x\left(\tau_{k}\right)=b_{k} x\left(\tau_{k}\right) .
\end{gathered}
$$

Introduce the following condition:

H4: $\quad q \in C\left(\mathbb{R}_{+}, \mathbb{R}_{+}\right)$.

Theorem 5: Let the following conditions hold:

1. Conditions $\mathrm{H} 1-\mathrm{H} 4$ are met.

2. $\quad \liminf _{k \rightarrow \infty} \int_{\tau_{k}-h}^{\tau_{k}} p(s) d s>\frac{1}{1+M}$.

Then equation (23) has no positive solutions.

Proof: Let $x(t)>0$ be a solution of (23) for $t \geq t_{0} \geq 0$. Integrate (23) from $\tau_{k}-h$ to $\tau_{k}$ $\left(\tau_{k}>t_{0}+h\right)$ and obtain

From (24) it follows that

$$
\begin{gathered}
x\left(\tau_{k}\right)-x\left(\tau_{k}-h\right)=\int_{\tau_{k}-h}^{\tau_{k}} p(s) x(s+h) d s \\
\quad+\sum_{\tau_{k}-h \leq \tau_{k}^{(s)} \leq \tau_{k}} b_{k}^{(s)} x\left(\tau_{k}^{(s)}\right)+\int_{\tau_{k}-h}^{\tau_{k}} q(s) d s .
\end{gathered}
$$

$$
x\left(\tau_{k}\right) \geq x\left(\tau_{k}+0\right) \int_{\tau_{k}-h}^{\tau_{k}} p(s) d s .
$$


From the last inequality we obtain that

$$
\int_{\tau_{k}-h}^{\tau_{k}} p(s) d s \leq \frac{x\left(\tau_{k}\right)}{x\left(\tau_{k}+0\right)} \leq \frac{1}{1+M}
$$

which contradicts condition 2 of Theorem 3 .

Introduce the following conditions:

H5: $\quad q \in C([0, \infty), \mathbb{R})$.

H6: There exists a function $v \in\left(C^{1}\left(\mathbb{R}_{+}, \mathbb{R}\right)\right.$ such that $v^{\prime}(t)=q(t), t \geq 0$.

H7: There exist constants $q_{1}$ and $q_{2}$ and two sequences $\left\{t_{i}^{\prime}\right\}_{1}^{\infty} \subset \mathbb{R}_{+}$and $\left\{t_{i}^{\prime \prime}\right\}_{1}^{\infty} \subset \mathbb{R}_{+}$with $\lim _{i \rightarrow \infty} t_{i}^{\prime}=\lim _{i \rightarrow \infty} t_{i}^{\prime \prime}=\infty$ and $v\left(t_{i}^{\prime}\right)=q_{1}, v\left(t_{i}^{\prime \prime}\right)=q_{2}, q_{1} \leq v(t) \leq q_{2}$.

Theorem 6: Let the following conditions hold:

1. Conditions $\mathrm{H} 1, \mathrm{H} 2, \mathrm{H} 5-\mathrm{H} 7$ are satisfied.

2. $b_{k} \geq 0, k \in \mathbb{N}$.

3. $\quad \limsup _{k \rightarrow \infty} \int_{\tau_{k}}^{\tau_{k}+h} p(s) d s>1$.

Then all solutions of equation (23) oscillate.

Proof: Let $x(t)>0$ be a solution of equation (23) for $t \geq t_{0}>0$.

Set

$$
z(t)=x(t)-v(t)+q_{1}
$$

Then from (23) we obtain that

$$
\begin{gathered}
z^{\prime}(t) \geq p(t) z(t+h), \\
\Delta z\left(\tau_{k}\right)=b_{k} z\left(\tau_{k}\right)+A_{k},
\end{gathered}
$$

where $A_{k}=b_{k} v\left(\tau_{k}\right)-b_{k} q_{1} \geq 0$.

1. Let the inequality (25) have a positive solution $z(t)$ for $t \geq t_{1} \geq t_{0}$. Integrate (25) from $\tau_{k}$ to $\tau_{k}+h, \tau_{k} \geq t_{1}$ and obtain that

$$
\begin{gathered}
z\left(\tau_{k}+h\right)-z\left(\tau_{k}+0\right) \geq z\left(\tau_{k}+h\right) \int_{\tau_{k}}^{\tau_{k}+h} p(s) d s \\
z\left(\tau_{k}+h\right)\left[\int_{\tau_{k}}^{\tau_{k}+h} p(s) d s-1\right] \leq 0 .
\end{gathered}
$$

The last inequality contradicts condition 3 of Theorem 6 .

2. Let $z(t)<0$ for $t \geq t_{1}$ be a solution of the inequality (25). Then,

$$
z\left(t_{i}^{\prime}\right)=x\left(t_{i}^{\prime}\right)-v\left(t_{i}^{\prime}\right)+q_{1}=x\left(t_{i}^{\prime}\right)>0, \quad t_{i}^{\prime} \geq t_{1} .
$$

Theorem 7: Let the following conditions hold: 
1. Conditions $\mathrm{H} 1-\mathrm{H} 3, \mathrm{H} 5-\mathrm{H} 7$ are met.

2. $\liminf _{k \rightarrow \infty} \int_{\tau_{k}-h}^{\tau_{k}} p(s) d s>\frac{1}{1+M}$.

Then all solutions of equation (23) oscillate.

Proof: Analogously to the proof of Theorem 6 we obtain (25).

Let $z(t)>0$ be a solution of (25) for $t \geq t_{1} \geq t_{0}$. Integrate (25) from $\tau_{k}-h$ to $\tau_{k}$ $\left(\tau_{k}>t_{1}+h\right)$ and obtain

$$
\begin{gathered}
z\left(\tau_{k}\right)-z\left(\tau_{k}-h\right) \geq z\left(\tau_{k}+0\right) \int_{\tau_{k}-h}^{\tau_{k}} p(s) d s \\
z\left(\tau_{k}\right) \geq\left[\left(1+b_{k}\right) z\left(\tau_{k}\right)+A_{k}\right] \int_{\tau_{k}-h}^{\tau_{k}} p(s) d s \\
z\left(\tau_{k}\right) \geq\left(1+b_{k}\right) z\left(\tau_{k}\right) \int_{\tau_{k}-h}^{\tau_{k}} p(s) d s .
\end{gathered}
$$

From the last inequality it follows that

$$
\int_{\tau_{k}-h}^{\tau_{k}} p(s) d s \leq \frac{1}{1+b_{k}} \leq \frac{1}{1+M},
$$

which contradicts condition 2 of Theorem 7 .

The case when $z(t)<0$ is considered analogously.

\section{Acknowledgements}

The present investigation was supported by the Bulgarian Ministry of Education, Science and Technologies under Grant MM--422.

\section{References}

[1] Gopalsamy, K. and Zhang, B.G., On delay differential equations with impulses, J. Math. Anal. Appl. 139:1 (1989), 110-122.

[2] Györi, I. and Ladas, G., Oscillation Theory of Delay Differential Equations with Applications, Clarendon Press, Oxford 1991.

[3] Ladde, G.S., Lakshmikantham, V. and Zhang, B.G., Oscillation Theory of Differential Equations with Deviating Arguments, Pure and Applied Mathematics 110, Marcel Dekker, 1987.

[4] Shevelo, V.N., Oscillations of Solutions of Differential Equations with Deviating Arguments, Naukova Dumka, Kiev 1978 (in Russian). 


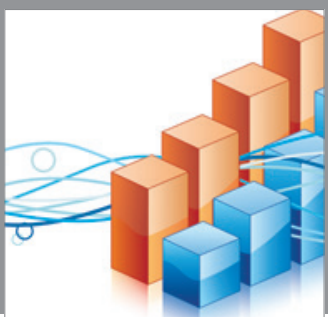

Advances in

Operations Research

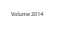

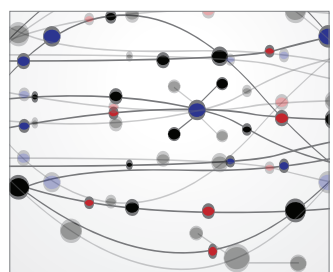

\section{The Scientific} World Journal
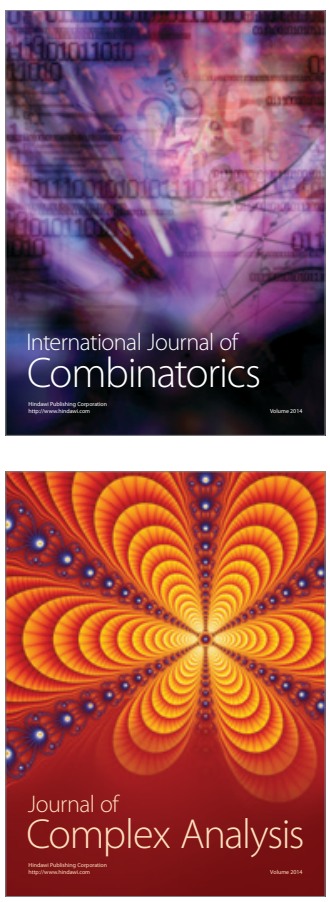

International Journal of

Mathematics and

Mathematical

Sciences
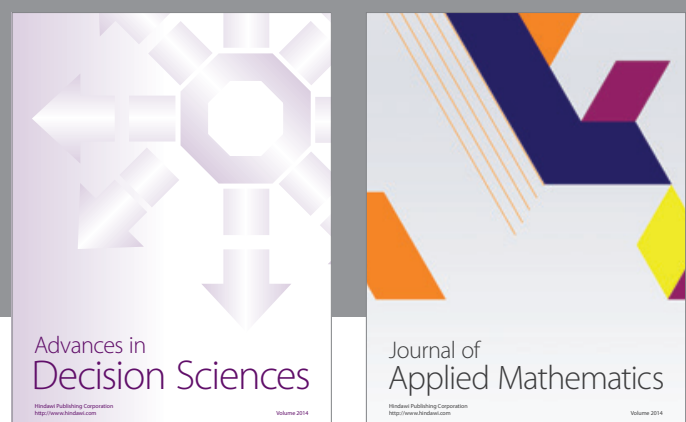

Journal of

Applied Mathematics
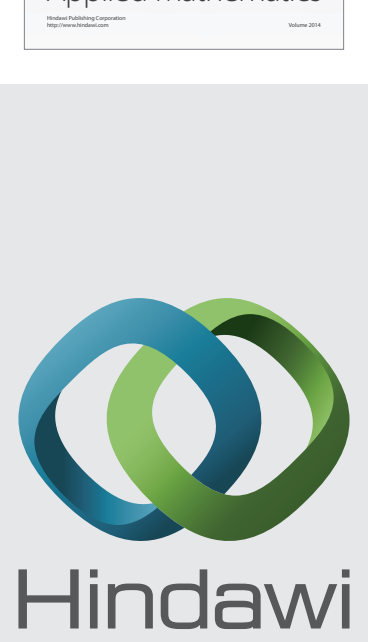

Submit your manuscripts at http://www.hindawi.com
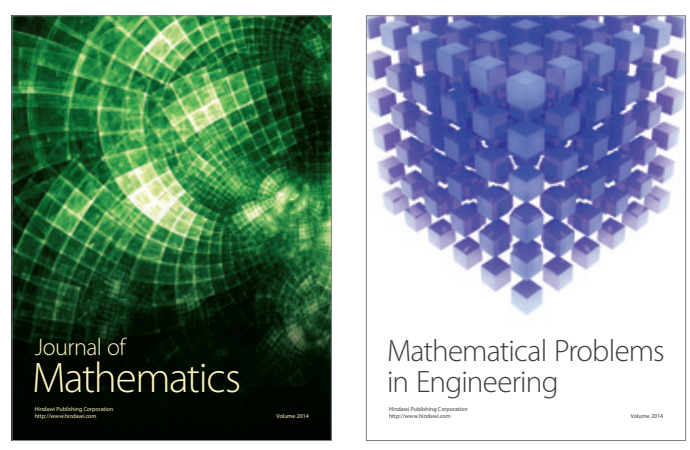

Mathematical Problems in Engineering
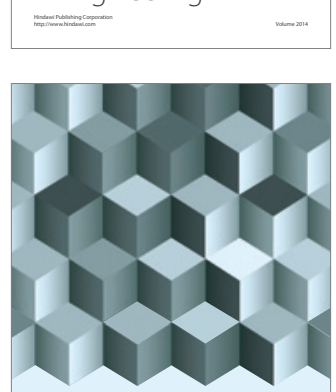

Journal of

Function Spaces
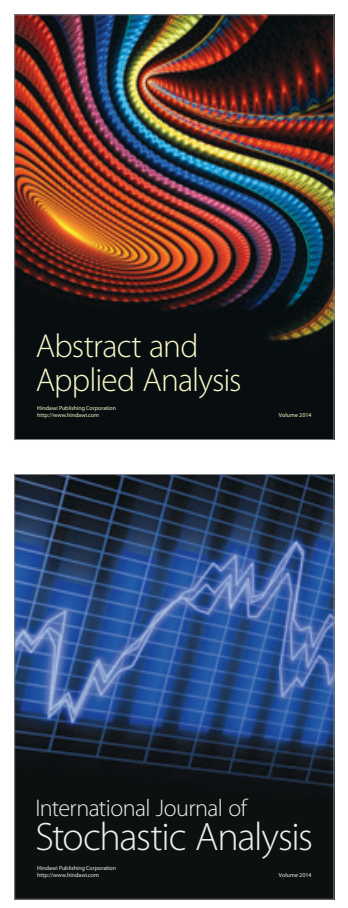

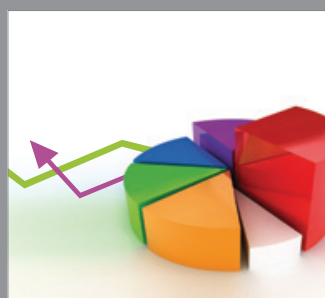

ournal of

Probability and Statistics

Promensencen
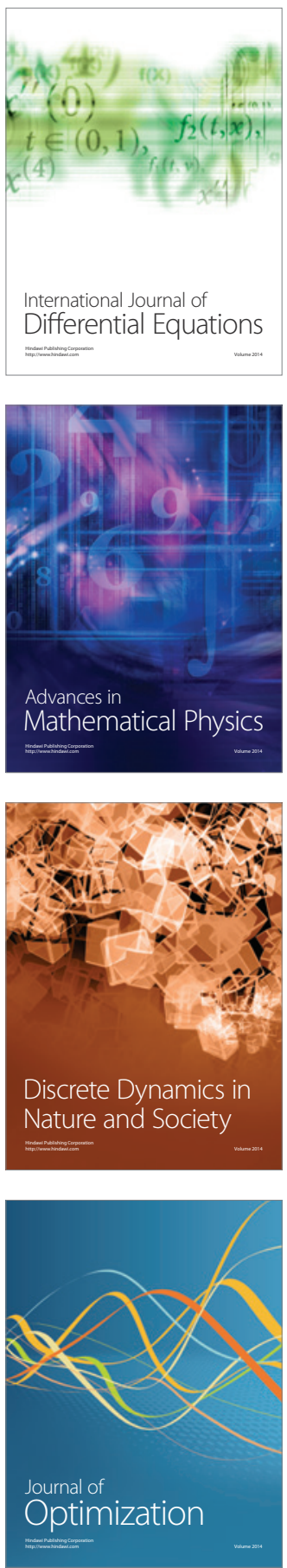